\title{
¿Qué fue la plaga de Atenas?
}

\author{
Jorge Dagnino S.
}

\section{What was the Plague of Athens?}

In the year 430 B.C., at the beginning of the second year of the Peloponessian War, a terrible epidemic fell upon Athens and the most populous cities in Attica. It would last for just over four years and it would kill 100.000 people, a quarter to a third of the population. We know about it through the masterly description made by Thucydides in his History of the Peloponnesian War. His narrative has withstood twenty five centuries due to its medical interest and, above all, its great dramatic force. The description of symptoms and signs, their evolution, and the consequences upon persons and moral and social order has captivated physicians, philologists and classical historians ever since. It has inspired literary works and hundreds of medical articles, with no agreement having been reached upon its cause or consequences, or if it is history or tragedy, or even if there is a single answer to these alternatives.

Key words: Plague, epidemics, Thucydides, history of Medicine.

Palabras clave: Plaga, epidemias, Tucídides, historia de la Medicina.
E sparta, que lideraba la liga del Peloponeso invadió Ática en el año 431 a.C iniciando así una brutal lucha fraticida que duraría veintisiete años y cambiaría irreversiblemente el mundo griego y la civilización antigua $^{1}$ (Figura 1). Apenas habían transcurrido cincuenta años desde las épicas batallas en las Termópilas, y luego en Salamina y Platea, donde los griegos entonces unidos enfrentaron y derrotaron al enorme ejército persa invasor en la segunda de las guerras médicas. Esta improbable victoria ante fuerzas inmensamente superiores en número fue seguida por décadas de gran desarrollo económico y cultural, particularmente en Atenas, con seminales avances en las artes, las ciencias y la medicina. Entre los líderes atenienses sobresale Pericles, cuya habilidad política y militar facilitaría el desarrollo

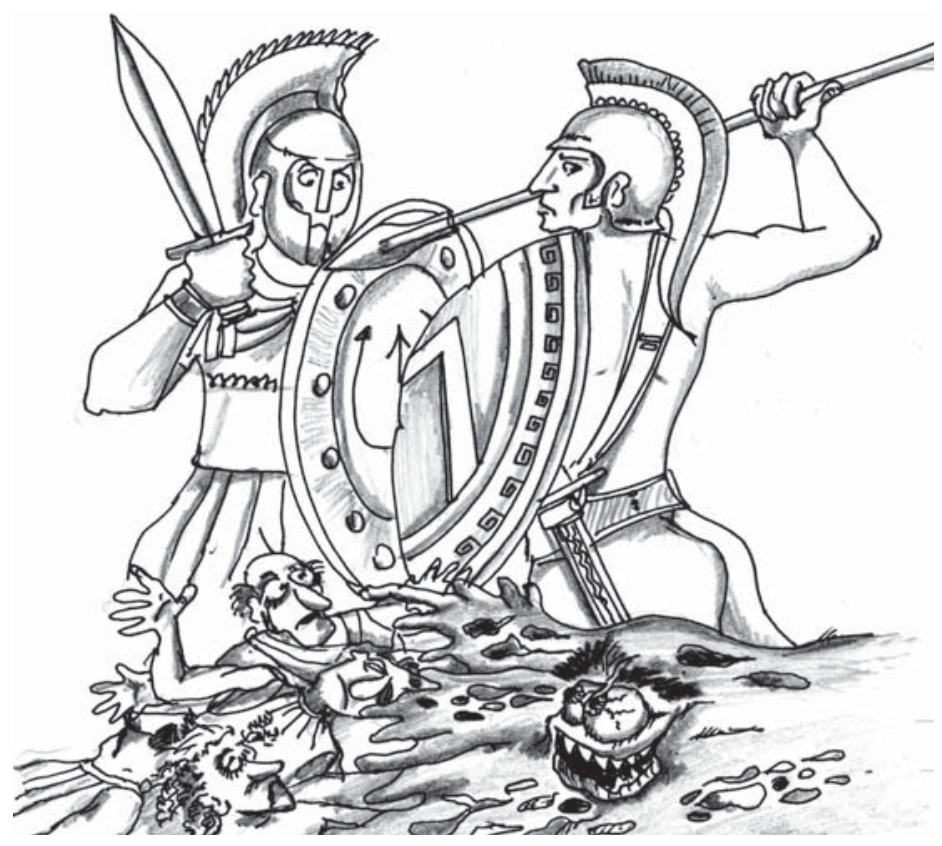

del imperio ateniense en el siglo que lleva su nombre. Al desatarse las hostilidades, Pericles, consciente de la superioridad de los hoplitas espartanos en el combate terrestre, impuso la estrategia de dejar los campos a merced del ejército invasor, protegiendo a la población en Atenas y su puerto, el Pireo. Estas ciudades estaban amuralladas y se encontraban unidas por un largo corredor defendido por las Murallas Largas que Temístocles había erigido al retirarse el ejército persa (Figura 2). Este complejo fortificado constituía una posición inexpugnable para las posibilidades bélicas de la época, mientras que la conexión marítima y la hegemonía ateniense en el mar aseguraban el abastecimiento indefinido de la población y permitían desarrollar ataques en las costas del territorio enemigo. A consecuencias de esta estrategia, la población de Atenas se había cuadruplicado con los refugiados, muchos de los cuales vivían hacinados en precarias chozas improvisadas, situación que creó en la capital del imperio y su puerto las condiciones ideales para el ataque de un enemigo mucho más peligroso que el ejército de Arquidamo, rey de Esparta ${ }^{2}$.

Al comenzar el segundo año de la guerra, en el inicio del verano de 430 a.C., una terrible epidemia cayó sobre Atenas y las ciudades más populosas de Ática, sin extenderse hacia la población o ejércitos del Peloponeso. Duraría cuatro años y moriría entre un cuarto y un tercio de la población; se dice que el mismo Pericles habría sido una de sus víctimas aun cuando su enfermedad fue arrastrada y se fue consumiendo lentamente ${ }^{1,2}$, imagen que no calza con el violento cuadro de la epidemia. Sabemos de ésta exclusivamente por la magistral descripción de Tucídides en el segundo libro de su Historia de la Guerra del Peloponeso, relato que sin duda es uno de los más impresionantes en la historia de la Medicina. Otras versiones de los hechos son muy posteriores, menos confiables y deben casi todo a la original: la de Diodorus Siculus en el siglo 1 a.C, de Plutarco en el siglo 1 d.C, o la de Galeno un siglo más tarde. El relato de la epidemia de Atenas está extrañamente ausente en otros textos de la época; en particular, no figura en ninguno de los tratados que conformarían más tarde el llamado Corpus hippocraticum ${ }^{3}$. La descripción de Tucídides es extraordinaria por su contenido y forma, y tiene tal fuerza que ha fascinado a historiadores, filólogos y médicos, quienes por siglos han buscado precisar las causas y las 
consecuencias de la epidemia. El relato ha sido también fuente de inspiración literaria en todos los tiempos; por nombrar algunos, el relato de Lucrecio en De rerum natura, el de Boccaccio en el prefacio del Decameron, Defoe en A Journal of the Plague Year, Manzoni en I Promessi Sposi, Camus en La Peste y Saramago en Ensayo sobre la Ceguera.

\section{Descripción de Tucídides}

El texto describe la catástrofe desde el punto de vista de la enfermedad y de sus consecuencias sobre la sociedad ateniense; en los dos ámbitos, es un modelo que aún hoy asombra por su riqueza, completitud y precisión. Médicamente, describe las circunstancias en que se produjo, el cuadro clínico con sus síntomas y signos y su evolución. Si bien en este artículo se favorece el uso de la palabra plaga, vale la pena decir que Tucídides no la usó

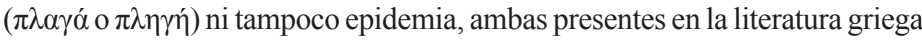

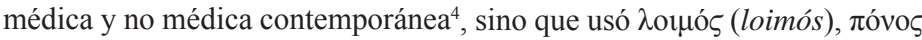
(pónos) y vóбoร (nósos). De estas tres, las dos primeras son hoy inusuales en el vocabulario médico: de loimós, como padecimiento, plaga o peste, derivan lemología o loimología, lemopira y epilema; de pónos, como trabajo o fatiga, ponos, cefaloponía; los derivados de nósos, como enfermedad o sufrimiento son más comunes: nosología, nosema, nosocomio, zoonosis ${ }^{5}$. La palabra plagá, como azote o golpe, se usaba en griego para denominar cualquier catástrofe; posteriormente, en la Edad Media, fue adquiriendo el significado de peste bubónica, particularmente en la literatura anglosajona. Peste o pestilencia derivan del latín y por ende son posteriores.

Tucídides deseaba dejar una descripción precisa para la posteridad, intención que declara explícitamente también para su obra completa. En relación a la plaga, dice que deja a cada lector, médico o profano, la opinión sobre su origen o causa y agrega: "por mi parte, simplemente la describiré por su naturaleza y explicaré sus síntomas por los que pueda ser reconocida por el estudioso si alguna vez se vuelve a presentar; esto lo puedo hacer mejor pues yo mismo sufrí el mal, y fui testigo de su actuar en el caso de otros" (T 2.48.3; la numeración corresponde al libro, capítulo y sección). Continúa diciendo que el padecimiento se habría iniciado en las tierras altas de Etiopía, desde allí descendió a Egipto y luego se extendió hacia al imperio persa. Habría aparecido en Lemnos, isla en el norte del mar Egeo a la salida del Helesponto, para llegar primero al puerto del Pireo y luego extenderse hacia Atenas, la ciudad alta. El relato sugiere que se extendió por las rutas comerciales, probablemente desde las costas del Mar Negro desde donde Atenas importaba trigo y otros productos. Es, por lo demás, lo que ha sucedido siempre en la diseminación de las epidemias a lo largo de la historia, siguiendo el comercio o la guerra.

Luego describe el cuadro clínico que aparece aquí en una traducción del autor desde la versión inglesa de principios del siglo XX ya citada ${ }^{6}$. Se ha tratado de conservar el ritmo aunque ello atente contra el estilo en español. El relato completo sobre la plaga va desde el capítulo 47, sección 3 al 54, sección 5 .

2.49.2 No hubo una causa ostensible; pero personas en buena salud eran repentinamente atacadas por violentos calores en la cabeza y enrojecimiento e inflamación de los ojos y las partes internas, como la garganta o la lengua, que se tornaban rojas y emitían un hálito anormal y fétido.

2.49.3 Estos síntomas eran seguidos de estornudos y ronquera, luego de lo cual el dolor llegaba pronto al pecho y producía una fuerte tos. Cuando se fijaba en el estómago lo indisponía; y seguían descargas

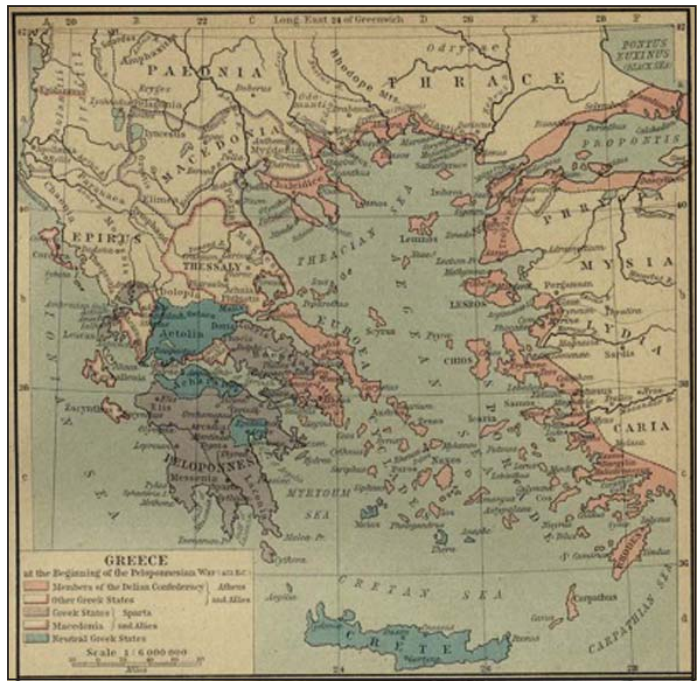

Figura 1. Grecia al comienzo de la guerra del Peloponeso.

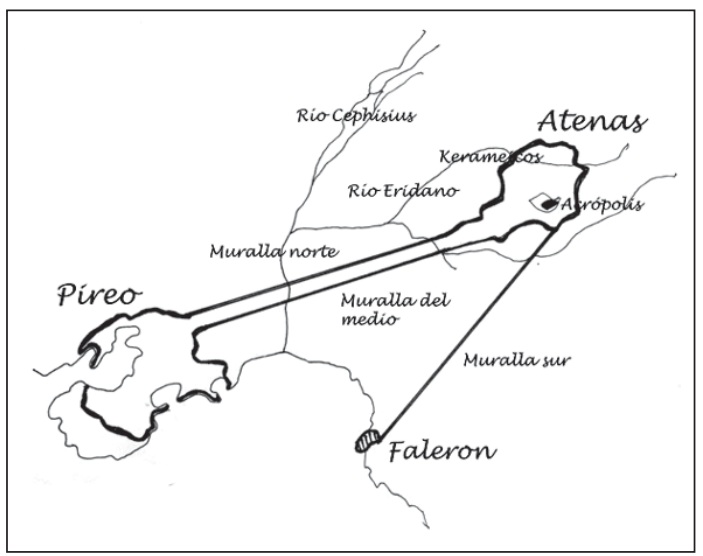

Figura 2. Atenas, el puerto del Pireo y las Murallas Largas de Temístocles.

de bilis de todos los tipos conocidos por los médicos, acompañadas de gran angustia.

2.49.4 En la mayoría de los casos seguían arcadas inefectivas, produciendo violentos espasmos, en algunos casos pronto después que cesaban los síntomas precedentes, en otros mucho después.

2.49.5 Externamente el cuerpo no estaba muy caliente al tacto, ni pálido en apariencia, sino que colorado, lívido y rompiendo en pequeñas pústulas y llagas. Pero internamente ardía tanto que el paciente no era capaz de soportar ropa o sábanas, incluso aquellas de la más liviana descripción, o en verdad de estar de cualquier otra manera que completamente desnudo. Lo que hubiesen querido más habría sido lanzarse al agua helada; como en efecto lo hicieron algunos de los enfermos descuidados, que se lanzaron a los estanques de agua lluvia en su agonía de sed inextinguible; aunque no hacía diferencia si tomaban poco o mucho.

2.49.6 Al lado de esto, la sensación miserable de no ser capaces de descansar o dormir jamás dejaba de atormentarlos. El cuerpo mientras tanto no se consumía mientras la enfermedad estaba en su apogeo, pero sorprendentemente se mantenía contra sus embates; de manera que cuando sucumbían a la inflamación interna, la mayoría de los casos al séptimo u octavo día, todavía tenían alguna fuerza en ellos. Pero 
si pasaban esta etapa de la enfermedad, y esta descendía más allá a los intestinos, induciendo allí una violenta ulceración acompañada de una intensa diarrea, esto traía una debilidad que era generalmente fatal.

2.49.7 Porque el desorden se asentaba primero en la cabeza, y seguía su curso desde allí a través de todo el cuerpo, e incluso cuando no resultaba mortal, aún así dejaba sus marcas en las extremidades.

2.49.8 Pues se asentaba en las partes pudendas, los dedos y ortejos y muchos escaparon con la pérdida de estos, algunos también con la de sus ojos. Aún otros también sufrían una pérdida total de la memoria en su primera recuperación y no se conocían a sí mismos ni a sus amigos.

Hay más antecedentes relevantes en la descripción de Tucídides. Dice que la aglomeración en la que vivían los refugiados agravó la miseria ya que fueron ellos los que más sufrieron, junto a los médicos y quienes cuidaban a los enfermos, pues adquirían la enfermedad con mayor frecuencia. Menciona que atacó a todos sin distinción y que quienes sobrevivían no volvían a tener la enfermedad o por lo menos no con resultado fatal; que las aves y animales que se alimentan de carne humana, a pesar de la cantidad de cadáveres disponibles, no se aproximaban a estos o bien morían luego de comer de ellos. Por último, describe con detalle la desesperanza que descendió sobre la población, y la disolución del orden social y moral como consecuencia de la gran mortandad y de la inefectividad de las plegarias, de los médicos y de las autoridades. Los muertos eran abandonados en cualquier lugar o bien los deudos aprovechaban las piras funerarias ajenas para arrojar a los muertos propios. Los enfermos eran abandonados a su suerte, y los desórdenes y crímenes se multiplicaban sin que el temor a los dioses o a la ley sirviesen de freno. Quienes tenían bienes o quienes se hacían de riquezas, por herencia o por no quedar dueños, derrochaban lo que tenían ante un futuro incierto. Más adelante se puede discernir que la plaga duró todo ese año y se mantuvo con casos esporádicos para reaparecer con fuerza en el verano siguiente y luego en el invierno de 427-426. Duraría poco más de cuatro años y perecerían unos 100.000 habitantes, entre un cuarto y un tercio de la población. Se estima esa cifra con base en el mismo Tucídides quien menciona que durante el sitio de Potidea, el ejército de Hagnon, que iba de refuerzo, contagió a quienes estaban sitiando la ciudad y que "murieron 1050 hoplitas de los 4000" (T2.58.3 $)^{6}$.

Antes de continuar la lectura, quizás el lector quiera ser uno de esos estudiosos a los que se refiere Tucídides y se aventure en formular un diagnóstico diferencial. A modo de resumen, en la Tabla 1 aparecen los síntomas y signos y otros antecedentes útiles que pueden ser deducidos de la descripción de Tucídides.

\section{Diagnóstico diferencial}

En los últimos cien años más de doscientos autores, -entre médicos, historiadores y filólogos clásicos-, unidos o por separado, han planteado con entusiasmo y defendido con mayor o menor elocuencia más de treinta posibles diagnósticos, la gran mayoría enfermedades infecciosas. Hay algunos que figuran recurrentemente en la literatura mientras que otros no han recibido mayor apoyo luego de la proposición inicial. Un recuento de ellos aparece en la Tabla 2. El que en alguna medida todos sean plausibles subraya desde el principio las dificultades para llegar a un consenso. De hecho, pareciera ser que en lo único en lo que se puede estar completamente de acuerdo es en que no hay acuerdo ${ }^{7}$.
Al ser el relato de Tucídides la única evidencia con la que contamos, es aparente que cualquier intento de identificación del cuadro depende de la exactitud de esa descripción, de la adecuada traducción de los términos que usó y de la interpretación médica de ellos ${ }^{7}$. Cada uno de estos factores ha sido objeto de análisis y controversias que enfrentan a filólogos clásicos, historiadores y médicos, unos y otros con dominio sobre áreas diferentes, cada una indispensable. Así, el filólogo o historiador clásico está limitado por la carencia de conocimientos médicos; el médico, por su desconocimiento de los matices del griego antiguo; y todos por la dificultad de hacerlo en términos y concepciones sobre salud y enfermedad separados por 25 siglos. $\mathrm{Al}$ respecto, hay dos consideraciones generales que vale la pena hacer. La primera es que al leer textos médicos antiguos es aconsejable intentar una

Tabla 1. Antecedentes epidemiológicos y cuadro clínico (Modificado de Cunha ${ }^{17}$ )

\begin{tabular}{lc} 
Semiología & Temporalidad \\
Comienzo brusco & Temprana \\
\hline Fiebre & $"$ \\
Ojos rojos & $"$ \\
Coriza y estornudos & $"$ \\
Faringe roja, ronquera, tos & $"$
\end{tabular}

Malestar precordial o epigástrico

Intermedia

Vómitos biliosos

Arcadas; espasmos o convulsiones

Piel lívida y roja o amarillenta; exantema

Ampollas o pápulas y llagas o bubones

Calor interno

Hiperestesia cutánea

Sed intensa

Agitación e insomnio

"

Diarrea y deshidratación

Gangrena distal o pérdida del uso

Ceguera

Amnesia o demencia

Muerte sin consunción al $7^{\circ}$ a $9^{a}$ día, excepto si diarrea

Tasa de ataque

"

"

"

Tardía

"

"

"

Mortalidad de los casos

Indeterminada (25-

$100 \%)$

$25 \%$

Edad, condición social o de salud

Contagio

Todos por igual

Inmunidad

Médicos y cuidadores

Epizootia

Total o parcial

Perros y ¿aves de rapiña?

Estacionalidad

Ausente

Duración

Cuatro años con brotes 
abstracción de los conocimientos actuales y aun de la imagen de la Medicina para reducir el riesgo de malinterpretar lo escrito ${ }^{8}$, especialmente a través de lo que ha sido denominada la "idealización de la medicina griega", el encontrar en ella el germen de conocimientos que asombran que pudiesen tener ${ }^{9}$. Un ejemplo de ello es el que la afirmación de Tucídides, de que quienes caían víctimas de la plaga con mayor frecuencia eran los médicos y quienes cuidaban a los enfermos, ha sido interpretada como la primera descripción de contagio en la literatura occidental, veinte siglos antes que Fracastoro. Sin embargo, es sólo una observación, sin una mayor elaboración o conceptualización. Algo similar sucede con el párrafo que describe la inmunidad y que vale la pena citar íntegro: "De aquellos que se recuperaban de la enfermedad, los enfermos y moribundos encontraron mayor compasión. Estos sabían por experiencia, y ya no temían mucho por ellos mismos, pues el mismo hombre nunca era atacado dos veces, nunca por lo menos fatalmente. Y esas personas no sólo recibían felicitaciones de otros si no que ellos mismos, en la euforia del momento, tenían la vana esperanza de que estaban seguros en el futuro ante cualquier enfermedad" (T.2.51.6).

Los aspectos más debatidos han sido la precisión con la que usa Tucídides la terminología médica y su conocimiento de las teorías médicas de la época. Hay quienes argumentan que Tucídides usaba con propiedad los términos médicos lo que permite deducir que conocía las doctrinas vigentes ${ }^{7}$. Al ser contemporáneo de Hipócrates, es probable se hayan conocido personal-

Tabla 2. Lista de posibles causas de la plaga de Atenas

Más discutidas

Tifus epidémico $(2,18,19,37)$

Fiebre tifoidea (29)

Viruela (20)

Sarampión $(7,14,17)$

Peste bubónica $(12,38)$

Otras

Ántrax

ATA (Alimentary Toxic Aleukia) (39)

Cólera

Dengue

Ébola

Ergotismo (40)

Erisipela

Escarlatina

Escorbuto

Fiebre de Lassa (41)

Influenza

Leptospirosis (43)

Malaria

Melioidosis (44)

Muermo (43)

Sífilis

Tularemia (43)

Combinación de enfermedades $(15,19)$

Fiebre amarilla y escorbuto

Fiebre tifoidea y disentería

Fiebre tifoidea y fiebre amarilla

Fiebre tifoidea y peste bubónica

Influenza e infección estáfilocóccica (42)

Enfermedades extintas (45)

Drama o Historia $(34,36)$ mente aun cuando no hay evidencia alguna de ello; por otro lado, los textos médicos constituían una buena proporción de los escritos disponibles para una persona culta. Otros opinan que muchos de los términos denominados médicos se encuentran en otras obras no médicas contemporáneas de manera que su conocimiento no necesariamente implica el dominio de su significado preciso o del valor médico del texto de Tucídides ${ }^{10}$.

Uno de los puntos más debatidos y en alguna medida manipulados, pues el diagnóstico depende en forma muy importante de la naturaleza del exantema, si es que fue eso lo que relata Tucídides, es el significado de la

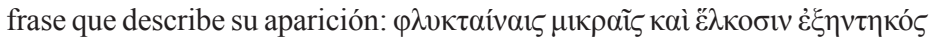
$(2.49 .5)^{11}$, en una transliteración, fluctaínais mikraís kai hélkesin exeenteekós, y traducido a vesículas o pústulas pequeñas y heridas rompiendo. La mayoría de los autores traducen $\varphi \lambda v \kappa \tau \alpha i ́ v \alpha 15$ como vesículas o ampollas, pero hay quienes lo leen como pápulas; en griego, el término deriva del verbo hervir y en el lenguaje corriente se refería a ampollas o manchas del pan o a ampollas en las manos de los remeros. Algo similar sucede con el substantivo que sigue, $\varepsilon \grave{\lambda} \kappa \circ$; generalmente interpretado como úlcera, herida o llaga, hay quienes, estirando la argumentación filológica, han postulado que el historiador podría estar describiendo bubones ${ }^{12}$.

También en la evolución del exantema hay diferentes interpretaciones posibles. Muchos han basado el diagnóstico en un exantema que se propaga desde la cabeza a las extremidades lo que ciertamente circunscribe las posibilidades diagnósticas. Sin embargo, no queda claro si Tucídides, cuando dice que la enfermedad cursa de la cabeza hacia abajo (T2.49.7), se refiere a los cambios cutáneos o al curso general de la enfermedad, puesto que el relato de Tucídides se puede ajustar a la concepción hipocrática sobre el flujo y fijación de la enfermedad ${ }^{13}$, y la frase que apunta a esta progresión está puesta a modo de resumen y separada de la descripción del exantema.

Hay otros ejemplos sobre las ambigüedades en la traducción que, aunque quizás tengan menos importancia en el diagnóstico diferencial,

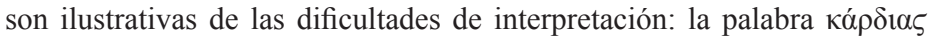
(kárdias) (T2.49.3) es interpretada como corazón ${ }^{7}$ o por estómago ${ }^{6}$; en Tucídides pareciera mejor esta última pues se da en el contexto de que la enfermedad va descendiendo desde el pecho hacia abajo y que, luego de

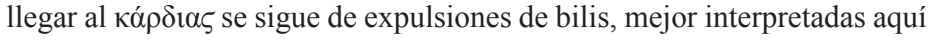
como vómitos biliosos; otros ejemplos son sangramiento o enrojecimiento, ceguera o daño ocular, arcadas o hipo, espasmos, calofríos o convulsiones, palidez o ictericia, amnesia o demencia, gangrena o pérdida del uso o incluso extirpación quirúrgica.

En el diagnóstico diferencial es importante distinguir entre una semiología compatible con una determinada enfermedad con aquella que es característica de ella, la que se viene a la mente con la sola mención del nombre de la enfermedad. En este sentido, puede decirse que el cuadro descrito por Tucídides es compatible con muchas enfermedades, como lo indica la multitud de diagnósticos propuestos, pero es característica de ninguna, como lo sugiere la falta de acuerdo en las muy buenas revisiones del tema ${ }^{14-21,46}$ o controversias de otras publicaciones ${ }^{22-24}$. En la Tabla 2 se agregan otras referencias a enfermedades específicas; la lista y las referencias no pretenden ser exhaustivas pues ya hace más de medio siglo las publicaciones sobre el tema alcanzaban un número inmanejable en un artículo de esta naturaleza ${ }^{7}$. Las etiologías más frecuentemente planteadas son peste bubónica, fiebre tifoidea, tifus epidémico, viruela y sarampión. Todos los autores discuten los signos y síntomas presentes, si calzan o no con una determinada enfermedad y ponen énfasis en aquellos característicos presentes y en aquellos llamativamente ausentes. Para explicar esta ausencia, -constreñidos por la aceptación general sobre la gran capacidad de observación, precisión y minuciosidad del relato de Tucídides-, se recurre a interpretaciones sobre 
el significado de los términos usados por Tucídides. Por ejemplo, el que no mencione las cicatrices de la viruela sobre todo si el mismo Tucídides fue víctima de ella; se ha inferido que con hélkos se puede plausiblemente entender cicatrices. Otro ejemplo, ya mencionado, para hacer dudar del diagnóstico de peste bubónica es que no hay mención de bubones, aunque era un término usado en la época. Otros síntomas y signos que faltan en un cuadro característico: diarrea, muerte por agotamiento o la mortandad de perros y aves carroñeras en viruela o sarampión; o la sintomatología respiratoria en el tifus epidémico o fiebre tifoidea. Quizás resumiendo las dificultades que enfrentan los estudiosos, dos revisiones recientes por un infectólogo llegan a conclusiones diferentes usando un método parecido: viruela o sarampión, y más bien este último, en $2004^{17}$ (vale la pena agregar aquí que el diagnóstico diferencial entre las dos enfermedades recién la vino a hacer Rhazes ${ }^{25}$ catorce siglos después de los acontecimientos relatados por Tucídides), y tifus epidémico en $2008^{18}$.

En la última década del siglo XX, se usó la modelación matemática epidemiológica para circunscribir las posibilidades diagnósticas. Para ello se incluyeron parámetros de epidemias conocidas que fueron contrastados con aquellos derivados de la descripción de Tucídides; el modelo contempla datos como la población total y su densidad, las tasas de ataque y de mortalidad, presencia o ausencia de inmunidad, velocidad de crecimiento y de desaparición. Por un proceso de eliminación, los autores concluyen que "la plaga de Atenas puede ser limitada a una enfermedad de reservorio (zoonótica o por vector) o bien a una enfermedad respiratoria que pueda asociarse con algún mecanismo inhabitual de persistencia. La primera categoría incluye tifus epidémico, enfermedades por arbovirus y peste bubónica; la segunda, viruela y, mucho menos probable, sarampión ${ }^{26,21}$.

En la misma década se empezó a describir la paleomicrobiología, técnica que ha permitido saldar controversias o simplemente confirmar la causa de diversas epidemias medievales ${ }^{27}$. El descubrimiento de una fosa común en los terrenos del muy antiguo cementerio Kerameikos (Figura 2), durante la excavación de una estación de metro mientras Atenas se preparaba para las olimpíadas del 2004, reveló los restos de unos 150 niños, mujeres y hombres, enterrados en forma progresivamente apresurada y desordenada, sin evidencia del cuidadoso ceremonial fúnebre como era la costumbre de los atenienses, y con cerámica votiva estimada del año 430 a.C. De la pulpa dentaria de tres osamentas se aisló ADN que fue identificado como Salmonella enterica serovar Typhi. Los autores concluyen que este podría ser el agente causal de la peste ${ }^{28-30}$. El hallazgo y sus conclusiones han sido controvertidos $^{31}$, y no han sido replicados hasta ahora. Las objeciones se centran en que la fiebre tifoidea era endémica en el Mediterráneo por lo que el hallazgo pudiese se colonización y no infección, en la metodología usada para procesar y secuenciar el ADN y en hacer una inferencia causal basada en una muestra de apenas tres dientes.

La mayoría de los autores comentados hacen fe en la exactitud del relato de Tucídides, argumentando que es poco probable que omitiese síntomas o signos cardinales. Esto hace pensar que la dificultad en calzar una enfermedad con los síntomas observados depende más bien de un exceso de ellos y no en su carencia. Uno de los problemas para discernir mejor este punto reside en el hecho que se sabe relativamente poco con certeza sobre la vida de Tucídides y sobre cómo escribió su historia. Además, como no hay mención sobre la plaga en otros textos contemporáneos no se pueden contrastar versiones. Tucídides habría nacido en 460 a.C., el mismo año que Hipócrates, por lo que tenía treinta años al comenzar la guerra. Tenía posesiones en Tracia que le daban un buen pasar y es probable que buena parte de su historia haya sido escrita en esas latitudes. Participó en la guerra como general, estrategos, pero fue castigado y exiliado cuando se le responsabilizó de la pérdida de Anfípolis, en las costas de Calcidice, al llegar tarde con refuerzos desde la vecina Tasos (Figura 1). No se sabe con certeza cómo escribió su historia ni tampoco el momento en que lo hizo ${ }^{2,32}$. Además de lo poco que él mismo cuenta, el resto son deducciones basadas en el lenguaje, la consistencia interna y la congruencia externa del relato. Se sabe, por ejemplo, que tomaba apuntes, que algunos pasajes los escribió poco después de los acontecimientos y otros cuando habían transcurrido veinte o más años; también, que fue haciendo revisiones a lo largo del tiempo e hizo cambios para acomodar una visión diferente a la inicial, ya sea porque el paso del tiempo cambió su comprensión de los acontecimientos o porque recabó nuevos antecedentes. Su relato termina abruptamente, en mitad de una frase, cuando todavía faltaban años para que terminase el conflicto aun cuando el mismo vivió varios años después de terminada la guerra. Es probable que el episodio de la plaga lo haya escrito poco después que el mismo Tucídides se recuperó de ella ${ }^{2}$ pero no se sabe si lo revisó posteriormente. Se puede aventurar que en todos esos años seguramente relató muchas veces lo ocurrido en Atenas, en conversaciones en torno al fuego o la mesa, y es posible que en cada recuento haya ido agregando detalles ocurridos en otro tiempo o lugar. Este proceso de creación o construcción de recuerdos falsos está bien documentado ${ }^{33}$. La concepción de enfermedad en la época, el uso de conceptos hipocráticos como el de las crisis en determinados días o el ya mencionado del flujo y fijación de la enfermedad son también argumentos a favor de una construcción del cuadro de la plaga que calza con la concepción griega de enfermedad.

Otra alternativa que debe ser planteada, aunque vaya en contra de lo que el mismo Tucídides expresa de su Historia, es que la epidemia, tal como fue narrada, no haya existido nunca y que pueda ser sólo fruto de su imaginación, y que haya mezclado signos y conductas observados en diferentes contextos, agrupándolos y describiéndolos como lo hizo para lograr un mejor efecto dramático $^{34}$. La descripción sin duda tiene una gran fuerza lo que ha llevado a algunos a decir que Tucídides concibió su obra más como una tragedia que como un relato factual y que la ubicó en su historia inmediatamente después del celebérrimo discurso fúnebre de Pericles para contrastar dos realidades atenienses muy opuestas: el vibrante discurso que arenga y rinde homenaje a los atenienses con las glorias alcanzadas por su cultura y su imperio contra la decadencia material y moral posterior, agudamente, por el azote de la plaga, y también aquella de más largo plazo que terminó con la derrota de Atenas y sus aliados ${ }^{35}$. Similares comentarios siguieron al Decamerón donde la también muy dramática descripción de Boccaccio de la peste en el prólogo contrasta con la bucólica reunión de los diez personajes y sus historias de disolución. Daniel Defoe en su A Journal of the Plague Year mezcló datos históricos fidedignos, como los mortality bills, con el relato ficticio del talabartero, testigo protagonista de la Gran Plaga de Londres de 1665. El relato de Defoe, al igual que el de Tucídides y de Boccaccio, describe los síntomas y signos, la mortandad, el abandono de vivos y muertos y el desorden social y moral; fue considerado por años y por muchos como el mejor relato histórico de la Gran Plaga ${ }^{36}$.

\section{Conclusión}

Así, a la pregunta del título tendríamos que responder que por la vía del diagnóstico diferencial clínico es probable nunca se sepa con certeza cuál fue el agente que causó la gran plaga de Atenas y que la paleomicrobiología es posiblemente la mejor esperanza para estrechar el cerco sobre la verdadera causa. La evidencia actual tampoco permite decir con certeza si el relato es historia o ficción. Hay también diversas opiniones sobre la importancia 
que la plaga de Atenas tuvo en el desenlace de la guerra y por ende en la evolución del mundo antiguo. Para algunos, la pérdida de un cuarto o más de la población, la muerte de líderes y especialmente la de Pericles, y el derrumbe del orden social y moral, contribuyeron en forma importante a la derrota de la liga de Delos. Los costos en vidas de la plaga sumados a los de la brutal guerra fueron enormes para el mundo griego: la población masculina de Atenas una década después de finalizado el conflicto era quizás la mitad de lo que era al iniciarse el la guerra. Sin embargo, "un año después del fin de la guerra Atenas había recuperado la democracia plena; dentro de una década, su flota, murallas e independencia; un cuarto de siglo después, la mayoría de sus antiguos aliados y restablecido su poder al punto que se puede hablar de un segundo imperio ateniense"1. Finalmente, si el objetivo de Tucídides fue el de posibilitar que otros pudiesen reconocer la plaga si es que llegase a volver, este claramente no se cumplió si la plaga ya volvió o, quizás, hay que esperar a que regrese para ver si se cumple. Si su objetivo fue horrorizar y conmover, sin duda que lo ha venido cumpliendo durante 25 siglos y es probable lo siga haciendo en el futuro.

\section{Resumen}

En el año 430 a.C., al iniciarse el segundo año de la guerra del Peloponeso, una terrible epidemia se desató en Atenas y en las ciudades más populosas de Ática. Duraría algo más de cuatro años y morirían unas 100.000 personas, un cuarto a un tercio de la población. Sabemos de ella a través de la magistral descripción que Tucídides hace en su Historia de la Guerra del Peloponeso. Su relato ha perdurado por 25 siglos por su interés médico y, sobre todo, por su gran fuerza dramática. La descripción de los síntomas y signos, su evolución y las consecuencias sobre las personas y sobre el orden social y moral ha cautivado a médicos, filólogos e historiadores. Ha inspirado obras literarias y cientos de artículos sobre la etiología de la plaga sin que hasta el momento exista acuerdo sobre qué fue, si es historia o tragedia, e incluso, si es que hay una respuesta única a estas alternativas.

\section{Referencias}

1.- Kagan D. The Peloponessian War. New York: Penguin; 2004.

2.- Gomme A W. A Historical Commentary on Thucydides. Oxford: Clarendon Press; 1986.

3.- Jouanna J. Hippocrates. Baltimore: Johns Hopkins University Press; 1999.

4.- Liddell H G, Scott R. A Greek-English Lexicon. Oxford: Clarendon; 1973.

5.- Quintana Cabanas J M. Raíces griegas del léxico castellano, científico y médico. Madrid: Dykinson; 1997.

6.- Thucydides. The Peloponnesian War. Trad. J. M. Dent. New York: E. P. Dutton; 1910.

7.- Page D L. Thucydides' description of the great plague of Athens. Class Q 1953; 3: 97-119.

8.- Jones W H S. General Introduction. En Hippocrates, Vol. I, Loeb Classical Library. Cambridge: Harvard University Press; 1995.

9.- Laín Entralgo P. La curación por la palabra en la Antigüedad Clásica. Madrid: Revista de Occidente, 1958.

10.- Parry A. The language of Thucydides' description of the plague. Bull Inst Class Stud 1969; 16: 106-18.

11.- Thucydides. Historiae in two volumes. Oxford, Oxford University Press. 1942. Consultado en http://www.perseus.tufts.edu/hopper/text?doc=Perseus:t ext:1999.01.0199. 2 de abril, 2011.
12.- Hooker E M. Buboes in Thucydides? J Hell Stud 1958; 78: 78-83.

13.- Craik E M. Thucydides on the plague: physiology of flux and fixation. Class Q 2001; 51: 102-8.

14.- Shrewsbury J D F. The plague of Athens. Bull Hist Med 1950; 24: 1-24.

15.- Longrigg J. The great plague of Athens. Hist Sci 1980; 18: 209-25.

16.- Retief F P, Cilliers L. The epidemic of Athens, 430-426 BC. S Afr Med J 1998; 88: 50-3.

17.- Cunha B A. The cause of the Plague of Athens: plague, typhoid, typhus, smallpox, or measles? Infect Dis Clin North Am 2004; 18: 29-43.

18.- Cunha C B, Cunha B A. Great plagues of the past and remaining questions. En Raoult D, Drancourt M. Paleomicrobiology: Past Human Infections. Heidelberg: Springer; 2008.

19.- Durack D T, Littman R J, Benítez M, Mackowiak P A. Hellenic holocaust: A historical clinico-pathologic conference. Am J Med 2000; 109: 391-7.

20.- Littman R J, Littman M L. The Athenian plague: Smallpox. Trans Am Philol Assoc 1969; 100: 261-5.

21.- Littman R J. The plague of Athens: Epidemiology and paleopathology. Mount Sinai J Med 2009; 76: 456-67.

22.- Holladay A J, Poole JCF. Thucydides and the plague: A further note. Class Q 1984; 34: 483-85.

23.- Holladay A J. New developments in the problem of the Athenian plague. Class Q 1988; 38: 247-50.

24.- Holladay A J. The Thucydides syndrome. Another view. N Engl J Med 1985; 313: 1170-2.

25.- Abú Becr Mohammed Ibn Zacaríyá Ar-Rází. A Treatise on the small-pox and measles. Traducción de WA. Greenhill. London: Sydenham Society; 1848. Birmingham: The Classics of Medicine Library. 1987.

26.- Morens D M, Littman R J. Epidemiology of the plague of Athens. Trans Am Philol Assoc 1992; 122: 271-304.

27.- Drancourt M, Raoult D. Molecular insights into the history of plague. Microbes and Infect 2002; 4: 102-9.

28.- Papagrigorakis M J, Synodinos P N, Yapijakis C. Ancient typhoid epidemic reveals possible ancestral strain of Salmonella enterica serovar Typhi. Infect Genet Evol 2007; 7: 126-7.

29.- Papagrigorakis M J, Yapijakis C, Synodinos P N, Baziotopoulou-Valavani. DNA examination of ancient pulp incriminates typhoid fever as a probable cause of the plague of Athens. Int J Infect Dis 2006; 10: 206-14.

30.- Papagrigoraquis M J, Yapijakis C, Synodinos P N, Baziotopoulou-Valavani E. Insufficient phylogenetic analysis may not exclude candidacy of typhoid fever as a probable cause of the plague of Athens (reply to Shapiro et al.). Int J Infect Dis 2006; 335-6.

31.- Shapiro B, Gilbert M T P. No proof that typhoid caused the plague of Athens. Int J Infect Dis 2006; 336.

32.- Prentice W K. How Thucydides wrote his history. Class Philol 1930; 25: 117-27.

33.- Hyman Jr, I E. Errors in autobiographical memory. Clin Psychol Rev 1998; 18: 933-47.

34.- Morgan T E. Plague or Poetry? Thucydides on the epidemic at Athens. Trans Am Philol Assoc 1994; 124: 197-209.

35.- Soupios M A. Impact of the plague in Ancient Greece. Infec Dis Clin North Am 2004; 18: 45-51.

36.- Rubincam C. Thucydides and Defoe: Two plague narratives. Int J Class Trad 2004; 11: 194-212.

37.- MacArthur W P. The Athenian plague: A medical note. Class Q 1954; 4: 171-4.

38.- Williams E W. The sickness at Athens. Greece \& Rome, Second Series 1957; 4: 98-103. 
39.- Bellemore J, Plant I M, Cunningham L M. Plague of Athens - Fungal poison? J Hist Med Allied Sci 1994; 49: 521-45

40.- Salway P, Dell W. Plague at Athens. Greece \& Rome, Second Series 1955; 2: $62-70$.

41.- Hopper J M H. An arenavirus and the plague of Athens. J Roy Soc Med 1992; 85: 350-1.

42.- Langmuir A D, Worthen T D, Solomon C G, et al. The Thucydides syndrome.
N Engl J Med 1985; 313: 1027-30

43.- Wylie J A H, Stubbs H W. The plague of Athens:430-428 B.C. Epidemic and epizoötic. Class Q 1983; 33: 6-11.

44.- David E M. The good and the bad dying indiscriminately. The Athenian plague reconsidered. The Pahros; Spring 2000; 14.

45.- Poole J C F, Holladay A J. Thucydides and the plague of Athens. Class Q 1979; 29: 282-300. 